\title{
MICRO/NANO FLOWS: VORTICITY GENERATION
}

\author{
TREVOR H. MOULDEN \\ The university of Tennessee Space Institute, USA
}

\begin{abstract}
Vortical structures have been observed to develop in electrically driven fluid motion at the micro/nano scale, but no coherent theory has been put foreword in the literature to explain such a development. The present paper gives several results in a theory based upon the classical field equations. In particular, it is shown that the origin of vorticity production resides in the applied electric field interacting with any ion concentration gradients present in the fluid as defined by the vorticity equation. This is in addition to any viscous layer vorticity diffusion that may also exist in the flow.

Keywords: Micro/nano Scale Flows, Maxwell Tensor, Vorticity Production.
\end{abstract}

\section{INTRODUCTORY REMARKS}

The equations derived for any theory associated with fluid mechanics, or with physics in general, are of no practical use in applications unless they possess a unique regular solution. Since there is no complete proof of these properties for the Navier-Stokes/ion concentration equations, they have to be assumed before the following analysis has any merit. The particular application of interest herein is to electrically driven flows in micro/nano scale devices. See Silber-Li, et al. [1] for background details on a specific case that has been explored experimentally and which provides the background to the theory discussed herein. The practical importance of this class of flows has been demonstrated in several situations of interest.

The theory of fluid mechanics adopts a so-called hylomorphic structure, $\mathcal{H}=\left\{\mathcal{B}, \mathcal{D}_{t}\right\}$, as the framework for the theory. Here $\mathcal{B}$ is the fluid body of interest and $\mathcal{D}_{t}$ is the spatial domain occupied by that fluid body at time $t \in \mathbb{T}$. Thus, property fields are associated with the body $\mathcal{B}$ but are written as functions over the spatial domain $\mathcal{D}_{t}$. These property fields include the fluid density and temperature as well as those associated with the electric field. The domain $\mathcal{D}_{t}$ could be a function of time as the fluid body moves across space $\mathrm{R}^{3}$. The present study is only concerned with the generation of vorticity within the flow field (of fixed geometry) and not with the creation of vortical structures. The former is a property of the field equations, the latter, a feature of the entire boundary value problem: vortical structures are flow geometry dependent. The appearance of the flow, to distinct observers, is also distinct (as studied in the Plato problem; see Moulden [2] for example).

As noted by Levich [3] early work on the interaction of (direct current) electric fields with particles suspended in fluid motion was carried out by F. F. Reyss at the start of the nineteenth century. On the other hand, the study of micro/nano scale flows is quite recent with Chang et al. [4] providing a survey of such flows (including the role of vortex motion in the flow field development). However, that vortex instability is of a different kind to the property of the vorticity field discussed herein. No detailed theoretical mechanism for the formation of vortical motion was provided in the study of Chang et al. [4] (but some of their cited references contain such a discussion); it is appropriate therefore, to discuss the mechanism involved in more detail. In fact, there is nothing more than the standard vorticity equation, enlarged to include the action of electric fields, at work in this model of vortical flow development. Vorticity, $\zeta(\mathrm{x}, t)$, evolution (see equation 1.3) is discussed in Section 3 below; indeed the present study is only concerned with this vorticity generation. 
At the spatial dimensions appropriate for the micro/nano scale devices of interest herein it is readily shown that the molecular mean free path of the fluid is of such relative magnitude as to render the continuum theory an adequate representation of the flow. Hence the adoption of the Navier- Stokes equations (augmented with the Maxwell equations for electric fields) is quite appropriate for a discussion of such fluid motion. It is also assumed that the flow field is sufficiently smooth within the device of interest that the curl of the field equations can be extracted to describe the vorticity field for all appropriate space and time scales.

The proof of uniqueness is not direct for the equations that define electrically driven flows in micro/nano scale fluid mechanics due to the very non-linear nature of these equations. In addition, this set of field equations are coupled together in a non-trivial way. It can be noted (see Mao et al. [5] and Park et al. [6] for example) that linearizion is often undertaken to discuss the flow field at these small scales. However, such an approximation is not appropriate for the discussion of vortical structures in the flow field. Herein an exact theory will be examined in some detail. Gurtin [7] gave a simple uniqueness theorem for the Navier Stokes equations when regularity was assumed and when the body force was not a function of other field variables. This result, as written by Gurtin [7], cannot be extended for the present case which includes direct coupling of the body force with the other field variables. The uniqueness problem is discussed in subsection 3.2 below.

Any discussion of a uniqueness theorem must include the ion conservation equations (adopting the Nernst-Planck flux, $\mathbf{J}(\alpha)$, to determine the net charge density $\rho_{e}(\mathrm{x}, t)$ ). Again, it must be assumed that regularity issues associated with the solution to the entire set of field equations can be addressed (but this topic is not considered herein).

The central issues for the present deliberations are the creation of vorticity, $\zeta(\mathrm{x}, t)$, and vortical motion, in micro/nano scale fluid motion. This creation can only be studied from within the vorticity equation: that is the curl of the linear momentum equation (see equation (1.3) below). This equation shows directly that the source of vorticity resides in the curl of the Lorentz force, $\mathbf{F} \equiv \rho_{e} \mathbf{E}$. Here, $\rho_{e}$ denotes the net charge density given as the sum: $\rho_{e}=F \Sigma\left(z^{a} c^{a}\right)$. In addition, $F$, denotes the so-called Faraday constant while $c^{a}$ is the concentration of the $a^{\text {th }}$ ion. Finally, $z^{\alpha}$ represents the corresponding ion valence. Section 1.4, below, discusses the Nernst-Planck flux, $\mathbf{J}(\alpha)$. However, it can be noted that the cited literature on this class of flow does not consider the vorticity evolution equation. Knowledge of the properties of the vorticity equation are essential for a complete understanding of the flow development in micro-nano scale devices.

\subsection{The Mass Invariance Constraint.}

Since the flow in micro/nano scale devices takes place at very low speeds, the fluid density can be taken to be constant. This constant density statement is also based upon the assumed uniformity of the fluid composition. In the experimental data presented in Silber-Li et al. [8], the fluid consisted of Borax solution at $p H$ 9.2. Under these conditions, the mass invariance constraint reduces to the simple statement:

$$
\operatorname{div}(\mathrm{v}) \equiv \frac{\partial v_{i}}{\partial x_{i}}=0
$$

and is assumed to hold throughout the entire flow field. Here, $\mathrm{v}(\mathrm{x}, t)$, denotes the local velocity vector of the fluid. It is also assumed that the addition of Borax does not cause significant 
local viscosity and density property variations within the fluid. In the experimental situations of practical interest, there would be a continual mass flux through the entire physical device. However, the mathematical model adopted herein will assume constant fluid mass. It is recognized, thereby, that the evolution of rotational motion can be studied without a detailed knowledge of the process by which vortical structures are formed within the physical flow device. It is the possible effects of the electric field on the global flow evolution that are of major interest in the present context.

\subsection{Consequences of the Linear Momentum Equation.}

The linear momentum equation for a constant density fluid is standard save for the nature of the stress field throughout the fluid. That is, the stress field provided by the fluid pressure and the (assumed) linear viscous fluid model must be augmented by the addition of the so-called Maxwell tensor $\mathrm{M}(\mathrm{x}, t)$ (see equation (2.1). below) that arises from the action of the electric field. Specifically:

$$
\frac{\partial v_{i}}{\partial t}+v_{j} \frac{\partial v_{i}}{\partial x_{j}}-v \frac{\partial^{2} v_{i}}{\partial x_{j} \partial x_{j}}=\frac{F_{i}}{\rho}-\frac{1}{\rho} \frac{\partial P}{\partial x_{i}}
$$

where the Lorentz force, $\mathbf{F}(\mathrm{x}, t)=\operatorname{div}(\mathbf{M})=\rho_{e} \mathbf{E}$, with net charge density $\rho_{e}(\mathrm{x}, t)=\varepsilon \operatorname{div}(\mathbf{E}) \equiv$ $F \Sigma\left(z{ }^{a} c^{a}\right)$ (with summation taken over all distinct ions present). Properties of the tensor $\mathbf{M}$ are considered in more detail in Section 2 below. The curl operator applied to equation (1.2) produces the vorticity diffusion equation:

$$
\frac{\partial \zeta_{i}}{\partial t}+v_{j} \frac{\partial \zeta_{i}}{\partial x_{j}}-v \frac{\partial^{2} \zeta_{i}}{\partial x_{j} \partial x_{j}}=\zeta_{j} \frac{\partial v_{i}}{\partial x_{j}}+\varepsilon_{i j k} \frac{1}{\rho} \frac{\partial F_{k}}{\partial x_{j}}
$$

with $\zeta_{i}(\mathrm{x}, t)=\varepsilon_{i j k} \partial v_{k} / \partial x_{j}$ being the vorticity vector where the divergence, $\operatorname{div}(\zeta)$, is identically zero: there being no sources or sinks of vorticity in the flow field. Indeed, the divergence of equation (1.3) vanishes identically for constant density fluid flow. However, the last term on the right of equation (1.3) shows that the curl of the Lorentz force is the driving mechanism for vorticity generation. This vorticity generation says nothing about the development of vortical structures in the flow field; which are, in significant part, related to the device geometry. It also does not fully address the vorticity generation in wall viscous layers. The vorticity vector also participates in the generation of the scalar enstrophy, $\eta(\mathrm{x}, t)=\langle\zeta, \zeta\rangle / 2$, which is given as:

$$
\frac{\partial \eta}{\partial t}+v_{j} \frac{\partial \eta}{\partial x_{j}}-v \frac{\partial^{2} \eta}{\partial x_{j} \partial x_{j}}=\zeta_{i} \zeta_{j} \frac{\partial v_{i}}{\partial x_{j}}-\frac{v}{2} \frac{\partial \zeta_{i}}{\partial x_{j}} \frac{\partial \zeta_{i}}{\partial x_{j}}+\varepsilon_{i j k} \frac{\zeta_{i}}{2 \rho} \frac{\partial F_{k}}{\partial x_{j}}
$$

and, again, is non-linear. Here, the vorticity vector interacts with the curl of the Lorentz force (or, equivalently the electric field) to hasten the evolution of the enstrophy. This action is not viscosity related and does not depend upon the fluid pressure, $P(\mathrm{x}, t)$. There is also viscous dissipation at work that tends to dampen that same enstrophy evolution. Evolution of both the helicity tensor, $\mathbf{H}=\zeta \otimes \mathbf{v}$, and its scalar counterpart $h=\langle\zeta, \mathbf{v}\rangle$, are discussed below in Section 3.1. 


\subsection{The Maxwell Equations.}

It is assumed that no magnetic fields are acting upon the device of interest. Hence the Maxwell field equations can be stated in the reduced form:

$$
\partial \mathbf{E} / \partial t=-\mathbf{j} / \varepsilon, \quad \operatorname{curl}(\mathbf{E})=0 ; \quad \operatorname{div}(\mathbf{E})=\rho_{e} / \varepsilon
$$

where, as above, $\mathbf{E}(\mathrm{x}, t)$ denotes the applied electric field, $\mathbf{j}(\mathrm{x}, t) \equiv \sigma \mathbf{E}$ the local current density and $\varepsilon$ the (assumed constant) permittivity of the fluid. Finally, $\sigma$ denotes the (scalar) conductivity of the fluid. The properties of these field equations are discussed in Korvetz [9] for example. In the above equation, $\rho_{e}=F \Sigma\left(z^{a} c^{a}\right)$, denotes the net charge density and controls the change in the electric field strength via equation (1.5c). Also, $z^{a}$ is the ion valence and $c^{a}$ the corresponding ion concentration. No existence or uniqueness results for the entire set of field equations will be provided herein. Indeed, such results are not complete for the classical Navier-Stokes component of the above system of equations.

\subsection{Ion Conservation.}

The theory written down above is not closed until the ion conservation equations are introduced. Let $\mathrm{J}(\alpha)(\mathrm{x}, t)$ denote the Nernst-Planck flux as a function of spacetime. The $c^{a}$ ion is conserved according to the statement:

$$
\frac{\partial c^{\alpha}}{\partial t}+\frac{\partial J_{i}(\alpha)}{\partial x_{i}}=0
$$

and, for each of the $\alpha$-components, $c^{a}(\mathrm{x}, t)$ is time invariant if $\operatorname{div}(\mathbf{J}(\alpha))$ vanishes identically. Finally, the components of the Nernst-Planck flux, $J_{i}(\alpha)$, are taken in the standard form:

$$
J_{i}(\alpha)=-D_{\alpha} \frac{\partial c^{\alpha}}{\partial x_{i}}+v_{i} c^{\alpha}+\mu_{e p} F z^{\alpha} c^{\alpha} E_{i}
$$

Note here that the Nernst-Planck flux is related to the velocity field via the, non-linear $\mathrm{v} c^{a}$, term in equation (1.7). In that equation, $D_{a}$ represents the $\alpha$-ion diffusion coefficient which, herein, is assumed to be constant. Also, $\mu_{e p}$ is the ionic mobility (again assumed to be constant).

Equations (1.1-1.7) define the electro-fluid mechanics of the problem under discussion. Several questions naturally arise: for example "does this set of equations have a solution and, if so, is that solution unique?" being the most fundamental. Subsection 3.2 below makes a few comments (but does not answer such questions in their totality; indeed they have not been answered for the basic Navier Stokes equations - see Galdi et al. [10] for a summary of what is known about that set of equations). The form of the $J_{i}(\alpha)$ term in equation (1.7) ensures that equation (1.6) is non-linear. Hence there is, from equations (1.6) and (1.7), the statement:

$$
\frac{d}{d t} c^{\alpha}=D_{\alpha} \frac{\partial^{2} c^{\alpha}}{\partial x_{i} \partial x_{i}}-\mu_{e p} F z^{\alpha} \frac{\partial}{\partial x_{i}}\left[E_{i} c^{\alpha}\right]
$$

for the specification of ion conservation. This (scalar) equation contains an interaction between the electric field, $\mathrm{E}(\mathrm{x}, t)$, and the concentration, $c^{a}$, of the $\alpha$-ion as a contribution to 
the evolution of $d c^{\alpha} / d t$. In addition, there is an interaction with the velocity field in that material derivative term. Without the electric field acting, there is only convective diffusion of each $c^{a}(\mathrm{x}, t)$ component (which is akin to the action defined by the Navier Stokes equations in the absence of body forces or pressure gradients).

It is assumed in what follows that the fluid is entirely enclosed in the device of interest so that no free surface boundary conditions are required.

\section{THE MAXWELL TENSOR, M(x, $t)$}

Since electric fields are a central consideration for the flows of interest herein, it is necessary to introduce the Maxwell tensor, along with the Maxwell equations, that define the electric field. No magnetic fields are considered in the present development but those fields could be added without undue toil. Let $\mathrm{M}(\mathrm{x}, t)$ denote the Maxwell stress tensor. In the absence of any magnetic field, $\mathrm{B}(\mathrm{x}, t)$, acting on the fluid, this stress tensor has the explicit form:

$$
\mathbf{M}=\varepsilon[\mathbf{E} \otimes \mathbf{E}-\langle\mathbf{E}, \mathbf{E}\rangle \mathbf{I} / 2]
$$

as generated by an electric field $\mathbf{E} \in \mathrm{R}^{3}$ applied to a conducting fluid. Here, the (assumed constant) quantity, $\varepsilon \in \mathbb{R}$, denotes the fluid permittivity. From equation (2.1), there is trace $(\mathbf{M})=-\varepsilon\langle\mathbf{E}, \mathbf{E}\rangle / 2$. The eigensystem of the tensor $\mathbf{M}$ relates to that of $\mathbf{E}$ as shown in subsection 2.1.

It follows directly from equation (2.1) that $\operatorname{div}(\mathbf{M})=\rho_{e} \mathbf{E}$ [with net charge density $\left.\rho_{e}=\varepsilon \operatorname{div}(\mathbf{E})\right]$ and defines the Lorentz force, $\mathbf{F}(\mathbf{x}, t)$, acting on the conducting fluid. This force acts in unison with the viscous force, $v \operatorname{div}\left(\mathbf{T}^{v}\right)$, where under the Stokes model of the linear viscous fluid there is:

$$
T_{i j}^{v}=\mu\left[\frac{\partial v_{i}}{\partial x_{j}}+\frac{\partial v_{j}}{\partial x_{i}}\right]-P \delta_{i j}
$$

an equation which defines the Cauchy stress tensor. Then $\operatorname{div}\left(\mathbf{T}^{v}\right)$ represents the viscous force defined by the Cauchy stresses. It is a basic assumption herein that the fluid adopted in the experiments is linearly viscous. Then, to this viscous stress must be appended the stress associated with the above mentioned Lorentz force $\mathbf{F}(\mathrm{x}, t)$. That is, for the conducting fluid, the viscous stress tensor, $\mathbf{T}^{v}$, must be extended to the form: $\mathbf{T}^{v}+\mathbf{M} \equiv \mathbf{T}$, say. Hence, there is the total stress tensor, $\mathbf{T}(\mathrm{x}, t)$, given in the form:

$$
T_{i j}=\mu\left[\frac{\partial v_{i}}{\partial x_{j}}+\frac{\partial v_{j}}{\partial x_{i}}\right]-P \delta_{i j}+\varepsilon\left[E_{i} E_{j}-E_{k} E_{k} \delta_{i j} / 2\right]
$$

In addition, the Lorentz force is given explicitly as: $\mathbf{F}=\rho_{e} \mathbf{E} \equiv \operatorname{div}(\mathbf{M})$ in terms of the Maxwell tensor. The issue of uniqueness is mentioned below in Section 3.2 but cannot be given in a complete and final form. The mathematical difficulties in the development of such a theory being similar to those associated with the Navier-Stokes equations (see Galdi et al. [10]). Section 3.2, below, makes additional comments on the properties of these equations. It is assumed that the present micro/nano scale flows are all laminar so that turbulence models are not required in the following discussion. 


\subsection{The Eigensystem of M.}

The eigensystem of the tensor $\mathbf{M}$ (from the definition $\mathbf{M} \mathbf{X}=\lambda \mathbf{X}$ ) can be given in the explicit form:

$$
\lambda_{1}=\varepsilon\langle\mathbf{E}, \mathbf{E}\rangle / 2 ; \quad \mathbf{X}_{1}=\operatorname{span}\{\mathbf{E}\}
$$

along with the repeated eigenvalue $\lambda_{2,3}=-\langle\mathbf{E}, \mathbf{E}\rangle / 2$ and with its associated eigenvectors $\mathbf{X}_{2,3}$ (which reside in $\operatorname{span}\{\mathbf{E}\}^{\perp}$ the orthogonal complement of $\mathbf{X}_{1}$ and can be made orthogonal in that subspace). The $\mathbf{X}_{1}$ eigenvector, residing in the space $\operatorname{span}\{\mathbf{E}\}$, has significance for the overall flow development.

Let $\mathbf{d}(\mathrm{x}, t)=\mathbf{M n}$ denote the Maxwell stress vector on the surface $\mathbf{S}$ (if $\mathbf{n}$ is the outward normal to that surface). From the definitions:

$$
\langle\mathbf{d}, \mathbf{n}\rangle=\varepsilon\left[\langle\mathbf{E}, \mathbf{n}\rangle^{2}-\langle\mathbf{E}, \mathbf{E}\rangle / 2\right]
$$

so there must be the constraint $\mathbf{d} \mathbb{\perp} \mathbf{n}$ unless the equality $\mathbf{E} \equiv \mathbf{0}$ holds (which, of course, implies that the Maxwell tensor vanishes). That is, the stress vector does not lie in the plane that is perpendicular to the one-dimensional subspace, $\operatorname{span}\{\mathbf{n}\}$. In fact it is found that:

$$
4|\mathbf{d}|^{2}+\varepsilon^{2}|\mathbf{E}|^{2}=3 \varepsilon^{2}\langle\mathbf{E}, \mathbf{n}\rangle|\mathbf{E}|^{2}
$$

Note that $|\mathbf{d}|^{2}$ is linearly related to $|\mathbf{E}|^{2}$, so that: $|\mathbf{d}| \equiv 0$ if either $|\mathbf{E}| \equiv 0$ or $\langle\mathbf{E}, \mathbf{n}\rangle=1 / 3$; equalities that lacks generality for real flow applications.

\subsection{Causality Issues}

The basic field equations, equations (1.2), (1.3), (1.4), are material evolutionary equations so that their solution represents the changes in initial data due to the boundary conditions imposed. As in Moulden [2], this causality can be expressed in terms of a Greens function. Thus, for a differential equation of the form $\mathcal{D}(\mathbf{y})=\mathbf{f}(\mathbf{x})$, there is explicitly:

$$
\mathbf{y}(x)=\int_{\mathcal{D}} \mathbf{f}(\mathbf{x}) G(\mathbf{x}, \xi) d \xi+\mathcal{H}(\mathbf{x})
$$

for the solution. Here $G(x, \xi)$ is the Green's function associated with the differential operator $\mathcal{D}$. Equation (2.4) shows that changes to the vector $\mathrm{y}(\mathrm{x}, t)$ are caused by two distinct factors:

a). The forcing function $\mathbf{f}(\mathbf{x})$ when weighted by the Green's function.

b). The structure of the function $\mathcal{H}(\mathbf{x})$ due to the initial and boundary conditions imposed.

and applies separately to each of the field equations listed above. Similarly, the uniqueness of simple turbulence models was discussed in Moulden [11].

A Reynolds decomposition can be applied to equation (2.4) so that turbulent flow can be discussed if, indeed, the need for such a study were to arise. Such is not, however, the present interest.

The structure of causality in the fluid mechanics of electrically driven flows is a separate issue and will not be discussed herein. Some introductory comments on causality, in a 
different context, were given in Moulden [2]. It can be noted, however, that the electric field defines the Lorentz force, $\mathbf{F}(\mathrm{x}, t)$, which enters both the linear momentum equation (1.2), and the vorticity equation (1.3). Hence, its effect on the entire flow development is significant. The vorticity equation is transparent to the pressure field but not to the curl of the Lorentz force which acts as a driving force for vorticity generation.

\subsection{A Comment.}

Leng [12] raises questions concerning the nature of the models adopted in both fluid dynamical theory and in experimental testing: in particular the hypothesis that fluids can be treated as continua rather than via a molecular model. It is, of course, this assumption that allows fluid properties to be treated as a continuous function over $\mathbf{x} \in \mathrm{R}^{3}$ at each time $t \in \mathrm{R}$. Is this question more of a concern at the micro-nano scale flows considered herein? Not so, since the experimental devices of interest are only two or three orders of magnitude smaller than classical wind tunnel models. Leng's point would be well taken by the idealist, but not by the pragmatic observers required in a scientific study. It does, however, fall in line with the writing of Cartwright [13] who expresses a certain measure of caution concerning the mathematical models used in science. The latter text does, in fact, question the value of the entire set of theories put forward in that science. Much of the arguments used against the notions adopted in science are, however, understood background for those involved in the creation of that science. Science studies models of natural events and compares the properties of those models with reality. In no sense are these models the physical reality (however well they may mimic that reality).

\section{THE VORTICITY EQUATION}

As above, start from the linear momentum equation (see equation (1.2)) and apply the curl operation to obtain the non-linear vorticity evolution equation (1.3). It is then direct that the curl of the Lorentz force $\mathbf{F}(\mathrm{x}, t)$, is the contribution to vorticity evolution due to the applied electric field. This electric field-induced vorticity field arises throughout the entire flow field and not just from the wall boundary conditions. While it is demanded that the velocity field vanish on a solid surface, there is no such constraint on the vorticity field. However, the helicity tensor, $\mathbf{H}(\mathbf{x}, t)=\zeta \otimes \mathbf{v}$, will vanish on such a solid surface as does the scalar helicity $h(\mathbf{x}, t)=\langle\zeta, \mathbf{v}\rangle$. Section 3.1 discusses these issues in more detail. The so-called viscous layer develops adjacent to solid walls and is one seat of the flow induced vorticity production that is the main concern herein. Equation (1.3) defined that vorticity evolution which also depends upon the curl of the Lorentz force, $\mathbf{F}(\mathbf{x}, t)$, as well as an interaction of the vorticity field with the velocity gradient. Some specific issues that are of interest in micro/nano scale fluid motion will be discussed below.

\subsection{Helicity Evolution}

As defined above, let $\mathbf{H}(\mathbf{x}, t)=\zeta \otimes \mathbf{v}$ be the helicity tensor and also let $\mathrm{h}(\mathbf{x}, t)=\operatorname{trace}(\mathbf{H}) \equiv\langle\zeta, \mathbf{v}\rangle$ be the corresponding scalar helicity. Then there is a pair of evolution equations

$$
\left[\frac{d}{d t}-v \frac{\partial^{2}}{\partial x_{k} \partial x_{k}}\right] \mathrm{h}=H_{k i} \frac{\partial v_{i}}{\partial x_{k}}-\frac{\zeta_{i}}{\rho} \frac{\partial P}{\partial x_{i}}-2 v \frac{\partial \zeta_{i}}{\partial x_{k}} \frac{\partial v_{i}}{\partial x_{k}}+\frac{\zeta_{i}}{\rho} \frac{\partial M_{i l}}{\partial x_{l}}+k
$$


and

$$
\left[\frac{d}{d t}-v \frac{\partial^{2}}{\partial x_{k} \partial x_{k}}\right] H_{i j}=H_{k j} \frac{\partial v_{i}}{\partial x_{k}}-\frac{\zeta_{i}}{\rho} \frac{\partial P}{\partial x_{j}}-2 v \frac{\partial \zeta_{i}}{\partial x_{k}} \frac{\partial v_{j}}{\partial x_{k}}+\frac{\zeta_{i}}{\rho} \frac{\partial M_{j k}}{\partial x_{k}}+K_{i j}
$$

for the evaluation of $\mathrm{h}(\mathrm{x}, t)$ and $\mathbf{H}(\mathrm{x}, t)$ respectively. In the above equations the quantities $\mathbf{k}(\mathrm{x}, t)$ and $\mathbf{K}(\mathrm{x}, t)$ are provided with the definitions:

$$
k=\varepsilon_{i k l} \frac{v_{i}}{\rho} \frac{\partial^{2} M_{l m}}{\partial x_{k} \partial x_{m}} \quad \text { and } \quad K_{i j}=\varepsilon_{i k l} \frac{v_{j}}{\rho} \frac{\partial^{2} M_{l m}}{\partial x_{k} \partial x_{m}}
$$

as the contribution from the Maxwell stresses, $\mathbf{M}(\mathrm{x}, t)$, interacting with the velocity field in a non-trivial way. As is appropriate, equations (3.1) and (3.2) also contain contributions from the vorticity, $\zeta(\mathrm{x}, t)$, interacting with the Maxwell stresses and certain other kinematic quantities, as well as the pressure gradient. As is familiar from classical fluid mechanics, the vorticity vector, $\zeta(\mathrm{x}, t)$ from equation (1.3), is not dependent upon the pressure field, $P(\mathrm{x}, t)$ but in equations (3.1) and (3.2) the pressure gradient does interact with the vorticity field to modify the helicity evolution. The above definitions for $\mathbf{k}(\mathrm{x}, t)$ and $\mathbf{K}(\mathrm{x}, t)$, which constitute the diffusive components of the helicity evolution, show that these quantities must vanish on solid surfaces. Of course, $h(\mathrm{x}, t)$ vanishes on a solid surface as do the components of $\mathbf{H}(\mathrm{x}, t)$ (and of the divergence $\partial H_{i j} / \partial x_{j}$ ).

\subsection{On Uniqueness}

The following question must always be considered before any discussion of fluid motion can commence: is a given flow field unique? That is, from a given initial state, is it possible (with the same boundary conditions) for the flow to evolve in two distinct ways? That is the practical question and, apart for certain specific flow geometries, the answer is negative. On the other hand, it can be noted that very little can be said theoretically in this context since a full theory of uniqueness for this type of non-linear system has not been written down. What is known can be briefly stated in the following terms:

1. The linear mass invariance equation (1.1) must have a unique solution for properly prescribed initial and boundary conditions. Nothing more need be said herein.

2. The situation for equation (1.2) is not trivial and a full theory of uniqueness is not yet available (see Galdi et al. [10] as noted above). It is assumed herein that a unique solution does, in fact, exist for all boundary conditions of interest in the applications of micro/nano scale flows.

3. Little appears to have been clarified in the literature about the mathematical structure of the non-linear equation (1.8). Similar difficulties to those encountered for the Navier Stokes equations prevail for that equation also. Since the existence/uniqueness theory for the Navier Stokes equations is not complete, and the velocity field appears in equation (1.8), very little can be added, at this point, about its uniqueness. However, the Lorenz force is proportional to the vector $\mathbf{E}$, which is defined by the Maxwell equations $(1.5 \mathrm{a}, \mathrm{b}, \mathrm{c})$ and is unique for a prescribed electric field, $\mathbf{j}(\mathrm{x}, t)$.

If there is any non-uniqueness present then the difficulty becomes one of determining which solution (if any) has physical meaning. An extension of uniqueness theorems for the Navier 
Stokes equations, to include a simple turbulence model, was given in Moulden [11]. But that theory was not sufficiently general to satisfy most practical needs and the inclusion of the more complex turbulence models adopted in the applications.

\subsection{Viscous Layer Theory}

Classical boundary layer theory can be extended to treat the present physical situation where the effects of electric fields are significant close to any solid surface (as discussed in Probstein [14]). Following that reference it can be noted that close to a solid surface there is an electroviscous layer that is characterized by the processes of electro-osmosis and electrophoresis. These effects are not the current interest and need not be discussed at this point. As the wall boundary conditions for velocity it is assumed that a no-slip velocity condition prevails. This no-slip boundary condition naturally introduces a vorticity field as part of the wall viscous layer. Equation (1.8) introduces its own wall visco-electric layer involving both the ion concentration $c^{a}(\mathrm{x}, t)$ and the velocity field $\mathbf{v}(\mathrm{x}, t)$. That is the electric field $\mathbf{E}(\mathrm{x}, t)$ is associated with both the velocity and ion concentration fields via equations (1.2) and (1.8) and hence features in both the velocity and electric wall viscous layers.

\subsection{Vorticity Evolution}

As noted above, the evolution of discrete vortical structures is strongly dependent upon the geometry of the device of interest. Mao et al. [5], Park et al. [6] and Silber-Li et al. [1] all describe vortical motion evolving in micro-nano scale devices. The vorticity field, defined by equation (1.3), gives rise to a distribution of scalar helicity, $h(\mathrm{x}, t)$ and the tensor $\mathbf{H}(\mathrm{x}, t)$, as shown in equations (3.1) and (3.2). By definition, the helicity vanishes on a solid surface where the velocity field is zero. The same is not true of the vorticity vector field (in general) since the velocity gradient normal to the wall is not zero (except at points of flow separation). Hence on any solid surface, where $\left.\mathrm{v}\right|_{\text {wall }} \equiv 0$, (which implies that both $\left.h\right|_{\text {wall }}=0$ and $\left.\mathbf{H}\right|_{\text {wall }} \equiv 0$ ) it follows that the equation (3.2) above reduces to:

$$
\left[\frac{\zeta_{i}}{\rho}\left(\frac{\partial P}{\partial x_{j}}-\frac{\partial M_{j l}}{\partial x_{l}}\right)+2 v \frac{\partial \zeta_{i}}{\partial x_{k}} \frac{\partial v_{j}}{\partial x_{k}}\right]_{\text {wall }}=0
$$

with a contraction to give the limit form for equation (3.1) as a constraint upon the wall pressure gradient written in terms of the vorticity field (all components of which do not vanish due to the wall boundary condition). These conditions, $h=0$ and $\mathbf{H} \equiv \mathbf{0}$, are trivial for irrotational flows but not for the general viscous flows of current interest. These conditions involve the pressure gradient that exists on the bounding solid surface.

\section{CONSEQUENCES AND DISCUSSION}

It would be naive to assume that the Plato problem has no role to play in the discussion of this class of flow since the observation of vortical motion is very observer location dependent. In what follows, it is assumed that there are multiple observers who have the capacity to share and assimilate each other's data that was obtained from a given micro-nano scale fluid motion. The results of this assimilation provides the information upon which the comments below are based. 
1. Vorticity evolution in the flow is a direct consequence of the basic fluid property, $v$, the kinematic viscosity coefficient, as characterized by the Reynolds number. In addition, the geometry of the bounding domain serves as a source of vorticity via the wall boundary condition for viscous flow.

2. The applied electric field also causes, via the $\operatorname{curl}(\mathbf{F})$ term in equation 1.3, the generation of an incremental vorticity field within the flow field. This vorticity field will extend into the interior of the flow as the flow field develops. Discrete vortical structures may then evolve in the flow field.

3. It is not directly evident that vortical structures play any major role in the flow field evolution as far as the basic operation of the micro- nano scale device is concerned. They do, however, add interest to the structure of the flow field.

4. Other issues have not been discussed herein: the importance of the composition of the fluid; its $P h$ value (for example). Also, changes to the geometry of the device has not been considered. Certainly, these are important practical issues for the generation of vorticity in this class of flows.

The extent to which the problems discussed in Constantine [15] are significant for this class of flows has not been clarified. One such question concerns the inviscid limit of the field equations. Equation (3.3) would then reduce to the requirement that the set of quantities: $\left.\left[\zeta_{i}\left(\partial P / \partial x_{j}\right)\right]\right|_{\text {wall }}$, must all vanish on a solid wall (for all $i$ and $j$ ). Since the pressure gradient need not vanish under such circumstances, and not all components of the vorticity need vanish, this set of conditions need not be satisfied. Hence, this demand for an inviscid flow limit is of no interest for the present deliberations and will be rejected (at least for the class of flows under discussion).

\section{FINAL REMARKS}

The above discussion is mainly addressed to the production of vorticity in electric field driven fluid mechanics at the micro/nano scale. In particular, it has been shown that the curl of the Lorentz force is the source term for that evolution of vorticity. Just because vorticity is present in the flow field it does not imply that distinct vortical structures will be generated in that same flow field: these represent two distinct flow field structures (with their own developmental conditions) and are very device-geometry dependent.

However, the essential question concerning the existence and uniqueness of solutions to these non-linear field equations (even without the presence of an applied electric field) has not been clarified. The above cited references give some overview of the difficulties involved. This lack of a theory does not, of course, limit practical applications of the physics involved in this class of fluid motion.

As a final comment note that numerical solutions of the field equations discussed above are required before practical applications can be modeled. Such numerical studies are discussed in some of the references that are cited above.

\section{ACKNOWLEDGMENT}

Part of this work was carried out in support of the experimental study undertaken by Professor Silber-Li, Zhan-Hua and her staff at the Laboratory for Nonlinear Mechanics, Institute of Mechanics, Chinese Academy of Science, Beijing, 100190, China (see References (1) and (8) below). The hospitality provided by Professor Silber-Li, and her staff, in Beijing, where the present study was initiated, is gratefully acknowledged. 


\section{REFERENCES}

[1] Silber-Li, Z-H, Zheng, X., Kong, G-P. \& Moulden, T.H., Vortices in Micro/Nano Channel flows. Advances in Fluid Mechanics, Vol. IX, eds. M. Rahman \& C.A. Brebia, WIT Press: England, pp. 533-545, 2012.

[2] Moulden, T.H., On Causal Structures in Fluid Mechanics. Proc. 31th ISTS Conference, Matsuyama, Japan, 2017.

[3] Levich, V.G., Physicochemical Hydrodynamics. Prentic Hall, Englewood Cliffs, NJ, 1962.

[4] Chang, H-C, Yossifon, G. \& Demekhin, E.A., Nanoscale electrokinetics and microvortices: how microhydrodynamics affects nanofluidic Ion flux. Annual Review of Fluid Mechanics, 44, pp. 401-426, 2012.

https://doi.org/10.1146/annurev-fluid-120710-101046

[5] Mao, M, Ghosal, S. \& Hu, G-H., Hydrodynamic flow in the vicinity of a nanopore induced by an applied coltage. Nanotechnology, 24, 245202, 2013.

https://doi.org/10.1088/0957-4484/24/24/245202

[6] Park, S.Y, Russo, C.J, Branton, D. \& Stone, H.A., Eddies in a bottleneck: an arbitrary Debye length theory for capillary electroosmosis. Journal of Colloid and Interface Science, 297, pp. 833-839, 2006.

https://doi.org/10.1016/j.jcis.2005.11.045

[7] Gurtin, M.E., An Introduction to Continuum Mechanics. Academic Press, New York, 1981.

[8] Silber-Li, Z-H, Zheng, X, Kong, G-P. \& Moulden, T.H., The impact of Ion Concentration and $\mathrm{pH}$ values on the Vortices in Micro/Nano Channel Flows. $\mu$ Flu12. Proceedings of the 3rd European Conference on Microfluidics, Heidelberg, 2012.

[9] Korvetz, A., Electromagnetic Theory. Oxford University Press, Oxford, 2000.

[10] Galdi, G.P., Heywood, J.G. \& Rannacher, R. (eds), Fundamental Directions in Mathematical Fluid Mechanics. Springer, Basel, 2000.

[11] Moulden, T.H., Uniqueness Theorems in Turbulence Models. Proc. 29th ISTS Conference, Nagoya, Japan, 2013.

[12] Leng, M., Mathematics and Reality. Oxford University Press, Oxford, 2010.

[13] Cartwright, N., How the Laws of Physics Lie. Oxford University Press, Oxford, 1983.

[14] Probstein, R.F., Physicochemical Hydrodynamics, 2nd edn., Wiley Interscience, Hoboken, NJ, 2003.

[15] Constantine, P., A few results and open problems regarding incompressible fluids. AMS Notices, 47, pp. 658-663, 1995. 